See discussions, stats, and author profiles for this publication at: https://www.researchgate.net/publication/4266333

\title{
GPS/TEC estimation with IONOLAB method
}

Conference Paper · July 2007

DOI: 10.1109/RAST.2007.4283998 · Source: IEEE Xplore

\section{CITATIONS}

3

4 authors:

Halil Nayir

ASELSAN Inc.

6 PUBLICATIONS 217 CITATIONS

SEE PROFILE

Orhan Arikan

Bilkent University

197 PUBLICATIONS 2,654 CITATIONS

SEE PROFILE
READS

245

F. Arikan

Hacettepe University

142 PUBLICATIONS 1,060 CITATIONS

SEE PROFILE

Cemil B. Erol

SMART Defence R\&D Inc.

32 PUBLICATIONS 376 CITATIONS

SEE PROFILE

Some of the authors of this publication are also working on these related projects:

Off-Grid Problem in Compressed Sensing View project

MODELING OF STRUCTURAL PROPERTIES OF ANOMALIES AND DISTURBANCES FOR THE IONOSPHERE OVER TURKEY View project 


\title{
GPS/TEC Estimation with IONOLAB Method
}

\author{
H. Nayir', F. Arıkan', O. Arıkan ${ }^{3}$, C.B. Erol ${ }^{4}$ \\ ${ }^{1}$ Aselsan Inc., hnayir $a$ mst.aselsan.com.tr \\ M. Akif Ersoy Mah., 16. Str. No:16, Yenimahalle, Ankara, 06370, TURKEY \\ ${ }^{2}$ Hacettepe University \\ Department of Electrical and Electronics Engineering, arikan $a$,hacettepe.edu.tr \\ Beytepe, Ankara, Turkey \\ ${ }^{3}$ Bilkent University \\ Department of Electrical and Electronics Engineering, oarikan@ee.bilkent.edu.tr \\ Bilkent, Ankara, Turkey \\ ${ }^{4}$ TUBITAK, UEKAE \\ Kavaklıdere, Ankara, Turkey, cemil.erol@iltaren.tubitak.gov.tr
}

\begin{abstract}
Total Electron Content (TEC) is a key variable to measure the ionospheric characteristics and disturbances. The Global Positioning System (GPS) can be used for TEC estimation making use of the recorded signals at the GPS receiver. Reg-Est method that is developed by F.Arıkan, C.B. Erol and O. Arlkan can be used to estimate high resolution, robust TEC values combining GPS measurements of $30 \mathrm{~s}$ resolution obtained from the satellites which are above the $10^{\circ}$ elevation limit. Using this method, it is possible to estimate TEC values for a whole day or a desired time period both for quiet and disturbed days of the ionosphere. Reg-Est provides robust TEC estimates for highlatitude, mid-latitude and equatorial stations. In this study, some important parameters of Reg-Est such as ionospheric thin shell height, weighting function and receiver-satellite biases are investigated. By incorporating the results of the investigation, Reg-Est algorithm is developed into IONOLAB method. Thin shell model height is an important parameter for Single Layer Ionosphere Model (SLIM). In this study, it is shown that IONOLAB provides reliable and robust TEC estimates independent of the choice of the maximum ionization height. Signals from the low elevation satellites are prone to multipath effects. In order to reduce the distortion due to multipath signals, the optimum weighting function is implemented in IONOLAB, minimizing the non-ionospheric noise effects. GPS receivers record both pseudorange and phase data of signals. IONOLAB can input absolute TEC computed from the pseudorange measurements or phase-corrected low-noise TEC. The TEC estimates for both of these inputs are in good accordance with each other. Thus, taking either pseoudorange or phase-corrected measurement data as input, high resolution, robust TEC estimates can be obtained from IONOLAB. Another important parameter for TEC estimation is satellite-receiver instrumental biases. The biases are the frequency dependent delays due to satellite and receiver hardware. In order to compute TEC, satellite and receiver biases should be removed from GPS measurements correctly. However, the proper procedure of how to include them in the TEC computation is generally vaguely defined. IONOLAB suggests a technique for inclusion of the hardware biases obtained from the web for TEC estimates that are consistent with the results from the IGS analysis centers.
\end{abstract}

\section{INTRODUCTION}

Ionosphere forms the most important atmospheric layer for $\mathrm{HF}$ and satellite communication systems. Ionosphere varies with time, frequency, and location. Total Electron Content (TEC) provides a convenient measure for observing the variability of the ionosphere and characterization of the distortion on radio signals. TEC is defined as the total number of free electrons along a ray path of $1 \mathrm{~m}^{2}$ cross section. TEC is closely related to solar and geomagnetic activities. TEC is measured in TECU units ( 1 TECU $\left.=10^{16} \mathrm{el} / \mathrm{m}^{2}\right)$. The Global Positioning System (GPS), due to its availability for civilian use in the last 10 years, provides a cost-effective alternative for estimating TEC through recorded signals at the GPS receiver. Although the ionospheric group delay or phase advance on the recorded GPS signals is a major source of positioning errors, these parameters can be used to compute TEC efficiently.

Reg-Est method developed by F.Arıkan, C.B. Erol and O. Arikan is new alternative for estimation of robust TEC by combining GPS measurements of 30 s resolution obtained from the satellites which are above the $10^{\circ}$ elevation limit [1], [2], "in press" [3]. The method is based on combining GPS measurements in least squares sense. An optional weighting function and median filter is also applied. The method is capable of deriving TEC estimates for a whole day or for a limited period within a day.

The ionospheric thin shell height, weighting function and the use of web based satellite-receiver instrumental biases in RegEst are the parameters that are investigated in this study. The choice of ionospheric thin shell height, appropriate weighting function that minimizes the non-ionospheric irregularities and different methods for incorporation of instrumental biases are studied in detail. The method for phase-corrected TEC is developed and used as an alternative for absolute TEC in RegEst. The proper choice of alternative are incorporated into RegEst and the new method is called as IONOLAB. 


\section{REG-EST PARAMETERS}

In previous studies, Reg-Est algorithm is tried for various days and stations. It is shown that the method produces robust TEC estimates for various stations for both quiet and disturbed days in studies [1],[2] and "in press" [3]. The results are also compared with IRI-2001 and IGS analysis centers results. It is shown that Reg-Est TEC estimates are in good accordance with various analysis centers. Using Reg-Est method, estimates are obtained at higher time resolution compared to IRI-2001 and IGS results. Therefore, Reg-Est provides an important alternative for tracking the sudden ionospheric irregularities and disturbances.

In this paper, Reg-Est is applied to a larger range of GPS stations from mid-latitude, high-latitude and equatorial regions as given in TABLE 1. The days are selected from quiet and disturbed days of October 2003. The list of quiet and disturbed days are available at Ionospheric Dispatch Center (IDCE) [8]. As provided in [8], 10 October is quiet, 27-28-29 October are positively disturbed, 30-31 October are negatively disturbed days. In the last days of October 2003, a major geomagnetic and solar storm caused severe ionospheric disturbances. Kp index rose up to 9 and Dst index fell as low as $-400 \mathrm{nT}$. In this section, the effect of ionospheric parameters such as ionospheric thin shell height, weighting function and satellitereceiver instrumental biases are studied.

TABLE 1

List of GPS recevier stations

\begin{tabular}{|c|c|c|c|}
\hline Receiver Station & Country & Latitude $^{\circ}$ & Longtitude $^{\circ}$ \\
\hline Ankara & Turkey & $39,53 \mathrm{~N}$ & $32,45 \mathrm{E}$ \\
\hline Brüksel & Belgium & $50,47 \mathrm{~N}$ & $4,21 \mathrm{E}$ \\
\hline Graz & Austria & $47,04 \mathrm{~N}$ & $15,29 \mathrm{E}$ \\
\hline Istanbul & Turkey & $41,06 \mathrm{~N}$ & $29,01 \mathrm{E}$ \\
\hline Zelenchukskaya & Russia & $43,17 \mathrm{~N}$ & $41,33 \mathrm{E}$ \\
\hline Arti & Russia & $56,25 \mathrm{~N}$ & $58,33 \mathrm{E}$ \\
\hline Kiruna & Sweden & $67,51 \mathrm{~N}$ & $20,58 \mathrm{E}$ \\
\hline Metsahovi & Finland & $60,13 \mathrm{~N}$ & $24,41 \mathrm{E}$ \\
\hline Petropavlosk & Russia & $53,04 \mathrm{~N}$ & $158,36 \mathrm{E}$ \\
\hline Lae & Papua New & $06,40 \mathrm{~S}$ & $146,59 \mathrm{E}$ \\
\hline Ganila & Philippines & $14,38 \mathrm{~N}$ & $121,04 \mathrm{E}$ \\
\hline Nanyang & Singapore & $01,20 \mathrm{~N}$ & $103,40 \mathrm{E}$ \\
\hline
\end{tabular}

\section{A. Inclusion of satellite and receiver instrumental biases}

Instrumental satellite and receiver biases are important parameters for TEC estimation. GPS measurements include both ionospheric delay and satellite-receiver instrumental biases. In order to estimate ionospheric TEC, these instrumental biases should be removed from measurements in an appropriate way. In the literature, there is no standard procedure for inclusion of satellite and receiver bias parameters in TEC estimation. In this study, two satellite and receiver bias inclusion methods are tried for Reg-Est. These methods are given in the following equations. In Method 1, the satellite and receiver instrumental biases are used in STEC computation as in Eq. (1) [6],[7],[14].
Bias inclusion method 1:

$$
\begin{aligned}
& \operatorname{STEC}_{u}{ }^{m}(n)=\frac{1}{A} \frac{f_{1}^{2} f_{2}^{2}}{f_{1}^{2}-f_{2}{ }^{2}}\left[P_{4, u}{ }^{m}(n)+c\left(D C B^{m}+D C B_{u}\right)\right] \\
& \operatorname{VTEC}_{u}{ }^{m}(n)=S T E C_{u}{ }^{m}(n) / M\left(\varepsilon^{m}(n)\right)
\end{aligned}
$$

where

$$
M\left(\varepsilon_{m}(n)\right)=\left[1-\left(\frac{R \cos \varepsilon_{m}(n)}{R+h}\right)^{2}\right]^{-1 / 2}
$$

In the above equations, $P_{4}$ is the geometry free linear combination of pseudorange values $\left(P_{4}=P_{2}-P_{1}\right)$. $A$ is constant which is equal to $40,3 \mathrm{~m}^{3} / \mathrm{s}^{2} . D C B_{m}$ and $D C B_{u}$ are the frequency dependent satellite and receiver instrumental biases, respectively. $m$ denotes satellite, $u$ denotes receiver and $n$ is the time sample. In Eq. (2), STEC is converted to VTEC using a mapping function that is given in Eq. (3). $M$ is the mapping function and $\varepsilon$ is the satellite elevation angle. In Method 1, instrumental biases are in time units (s).

Method 2 includes satellite and receiver biases in VTEC computation. The biases are added in TECU units [1],[2] as shown below in Eq. (5).

$$
\begin{aligned}
& \text { Bias inclusion method 2: } \\
& \operatorname{STEC}_{u}{ }^{m}(n)=\frac{1}{A} \frac{f_{1}^{2} f_{2}^{2}}{f_{1}^{2}-f_{2}^{2}}\left[P_{4, u}{ }^{m}(n)\right] \\
& \operatorname{VTEC}_{u}{ }^{m}(n)=\operatorname{STEC}_{u}{ }^{m}(n) / M\left(\varepsilon^{m}(n)\right)+b^{m}+b_{u}
\end{aligned}
$$

Bias inclusion Method 1 and 2 are used in the computation of STEC and VTEC in preprocessing of input data for Reg-Est method for stations given TABLE 1 . The instrumantal biases are available in IONEX files of IGS analysis centers [12]. As an example, results for Petropavlovsk 12.10.2003 is given in Fig. 1. In Fig. 1, solid line and dashed line display the Reg-Est TEC estimates with bias inclusion method 1 and method 2, respectively. TEC estimates of various IGS analysis centers are also provided in Fig. 1. These TEC maps are obtained from [12]. In Fig. 1, JPL, CODE, ESA/ESOC, UPC estimates are displayed with diamond, square, circle and triangle symbols, respectively. As can be observed from Fig. 1 that the TEC estimates from Method 1 is very close to the results of CODE and estimates from both methods are in very good accordance.

Reg-Est estimates using both bias inclusion methods are compared with results of other analysis centers in by using $D_{l}$, $D_{2}$, and $D_{3}$ defined below. $\boldsymbol{x}_{b 1}$, and $\boldsymbol{x}_{b 2}$ are TEC estimation results of Reg-Est using method 1 and method 2 respectively. $x_{C O D E}$ represents the results of CODE analysis center. $N$ is the total number of GPS recordings for 24 hour period. In TABLE 2 computed TEC differences are listed for various days and stations. In general, $D_{2}$ results are smaller when compared to $D_{3}$. Thus, including instrumental biases as in Method 1 gives TEC estimation results closer to CODE analysis center. 


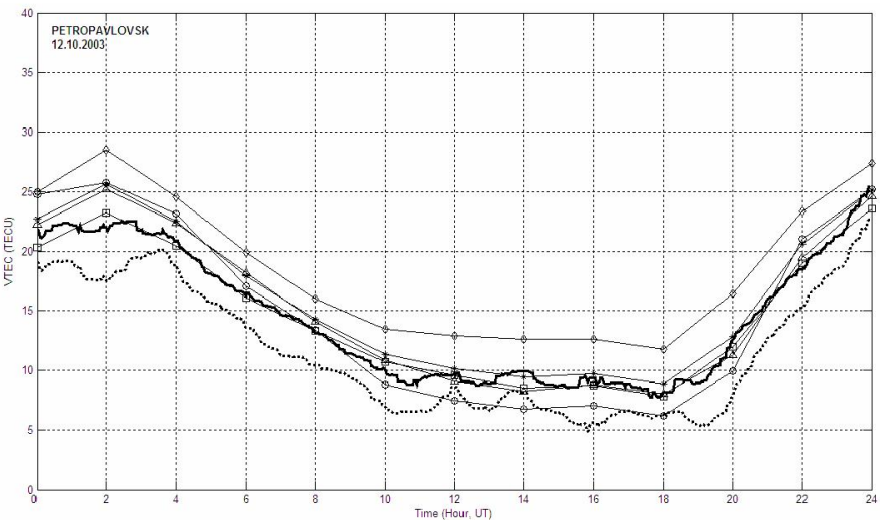

Fig. 1. Reg-Est TEC estimates obtained by applying method 1 and method 2 bias inclusion methods for Petropavlovsk 12.10.2003 (quiet day).

$$
\begin{gathered}
D_{1}=\frac{\sum_{n=1}^{N}\left|\boldsymbol{x}_{b 1}-\boldsymbol{x}_{b 2}\right|^{2}}{\sum_{n=1}^{N}\left|\boldsymbol{x}_{b 1}\right|^{2}} \\
D_{2}=\frac{\sum_{n=1}^{N}\left|\boldsymbol{x}_{b 1}-\boldsymbol{x}_{C O D E}\right|^{2}}{\sum_{n=1}^{N}\left|\boldsymbol{x}_{b 1}\right|^{2}} \\
D_{3}=\frac{\sum_{n=1}^{N}\left|\boldsymbol{x}_{b 2}-\boldsymbol{x}_{C O D E}\right|^{2}}{\sum_{n=1}^{N}\left|\boldsymbol{x}_{b 2}\right|^{2}}
\end{gathered}
$$

TABLE 2

Reg-Est TEC estimation differences obtained using different bias inclusion methods.

\begin{tabular}{|c|c|c|c|c|}
\hline Receiver Station & Day & $D_{1}$ & $D_{2}$ & $D_{3}$ \\
\hline Zelenchukskaya & Oct 12,2003 & $1.17 \times 10^{-2}$ & $2.85 \times 10^{-3}$ & $1.48 \times 10^{-2}$ \\
\hline Graz & Oct 31,2003 & $8.71 \times 10^{-3}$ & $1.73 \times 10^{-2}$ & $2.03 \times 10^{-2}$ \\
\hline Arti & Oct 10,2003 & $6.72 \times 10^{-2}$ & $4.21 \times 10^{-3}$ & $9.80 \times 10^{-2}$ \\
\hline Petropavlovsk & Oct 29,2003 & $1.81 \times 10^{-2}$ & $5.98 \times 10^{-3}$ & $4.17 \times 10^{-2}$ \\
\hline Nanyang & Oct 12,2003 & $1.27 \times 10^{-4}$ & $5.12 \times 10^{-3}$ & $5.27 \times 10^{-3}$ \\
\hline Lae & Oct 28,2003 & $8.94 \times 10^{-3}$ & $3.53 \times 10^{-2}$ & $7.23 \times 10^{-2}$ \\
\hline
\end{tabular}

Although using both bias inclusion methods in Reg-Est gives reasonable TEC estimates, bias inclusion Method 1 results are closer to IGS analysis centers' estimates compared to Method 2. Since using instrumental biases in STEC computation is more suitable for the model for GPS observation equations, Method 1 will be used in IONOLAB for inclusion of instrumental biases.

\section{B. Computation of Carrier Phase-Corrected VTEC}

Theoreticaly TEC can be computed using pseudorange data, carrier phase data or using combination of both [1]. Using pseudorange in TEC computation is simple and robust [1].
Pseudorange measurements are more noisy compared to carrier phase measurements. Using only carrier phase data in TEC computation is difficult because of initial phase ambiguity and cycle slips. Third method is to use both pseudorange and phase measurements to overcome phase ambiguity and cycle slip problems. These methods are discussed in various studies such as [5],[7],[9],[10],[14]. Previously, only the absolute TEC was used as an input to Reg-Est. For IONOLAB, the measurement input range is enlarged to include the phase-corrected measurements. Carrier phase measurements are levelled using pseudorange measurements to eliminate phase ambiguity. The levelling process is based on computing a baseline $(B)$ for each connected arc of phase measurements. Then, the computed baseline is used in STEC computation as in Eq. (10).

$$
\begin{gathered}
B^{m}=\frac{1}{N_{m e}} \sum_{n_{m e}=1}^{N_{m e}} P_{4, u}{ }^{m}\left(n_{m e}\right)-L_{4, u}{ }^{m}\left(n_{m e}\right) \\
\operatorname{STEC}_{u}{ }^{m}(n)=\frac{1}{A} \frac{f_{1}^{2} f_{2}^{2}}{f_{1}^{2}-f_{2}^{2}}\left[L_{4, u}{ }^{m}(n)+B^{m}+c\left(D C B^{m}+D C B_{u}\right)\right]
\end{gathered}
$$

Fig. 2 provides an example of the comparison of Reg-Est estimates obtained using pseudorange and carrier phase data. In Fig. 2.a., solid line and dotted line denote estimates obtained using carrier phase data and pseudorange data in Reg-Est, respectively. In Fig. 2.b., Reg-Est estimates are compared with the TEC estimates of IGS analysis centers. JPL, CODE, ESA/ESOC, UPC estimates are displayed with diamond, square, circle and triangle symbols, respectively. As can be observed from Fig. 2.b. that, using either pseudorange or carrier phase data as input, Reg-Est produces consistent TEC estimation results with IGS analysis centers especially with JPL and CODE. Therefore, IONOLAB can use both absolute TEC and phase-corrected TEC as input.
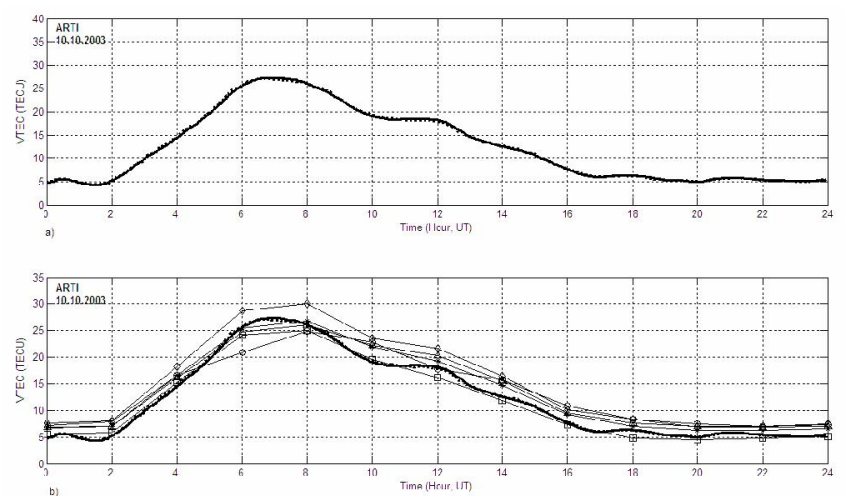

Fig. 2. Comparison of Reg-Est TEC estimates using pseudorange and carrier phase data, Arti 10.10.2003 (quiet day).

Detailed comparison of pseudorange and phase derived RegEst estimates with other analysis centers is done by computing normalized TEC differences as in equations (11) through (13), where $\boldsymbol{x}_{p r}$ and $\boldsymbol{x}_{p h}$ are Reg-Est estimates obtained using pseudorange and carrier phase data respectively. $\boldsymbol{x}_{J P L}$ and $\boldsymbol{x}_{C O D E}$ denotes the TEC estimates of JPL and CODE, respectively. The comparison results are 
given in TABLE 3 for various days and stations.

$$
\begin{aligned}
& D_{4}=\frac{\sum_{n=1}^{N}\left|\boldsymbol{x}_{p r}-\boldsymbol{x}_{p h}\right|^{2}}{\sum_{n=1}^{N}\left|\boldsymbol{x}_{p r}\right|^{2}} \\
& D_{5}=\frac{\sum_{n=1}^{N}\left|\boldsymbol{x}_{p r}-\boldsymbol{x}_{J P L}\right|^{2}}{\sum_{n=1}^{N}\left|\boldsymbol{x}_{p r}\right|^{2}} \\
& D_{6}=\frac{\sum_{n=1}^{N}\left|\boldsymbol{x}_{p h}-\boldsymbol{x}_{J P L}\right|^{2}}{\sum_{n=1}^{N}\left|\boldsymbol{x}_{p h}\right|^{2}}
\end{aligned}
$$

TABLE 3

Reg-Est TEC estimation differences obtained using pseudorange or carrier phase data.

\begin{tabular}{|c|c|c|c|c|}
\hline Receiver Station & Day & $D_{4}$ & $D_{5}$ & $D_{6}$ \\
\hline Ankara & Oct 31,2003 & $1.75 \times 10^{-4}$ & $1,30 \times 10^{-3}$ & $1.93 \times 10^{-3}$ \\
\hline Graz & Oct 10,2003 & $8.20 \times 10^{-4}$ & $3.20 \times 10^{-2}$ & $3.21 \times 10^{-2}$ \\
\hline Zelenchukskaya & Oct 29,2003 & $2.68 \times 10^{-4}$ & $1.99 \times 10^{-3}$ & $1.49 \times 10^{-3}$ \\
\hline Petropavlovsk & Oct 31,2003 & $5.07 \times 10^{-4}$ & $2.87 \times 10^{-3}$ & $2.68 \times 10^{-3}$ \\
\hline Arti & Oct 10,2003 & $2.29 \times 10^{-3}$ & $4.53 \times 10^{-2}$ & $4.59 \times 10^{-2}$ \\
\hline Kiruna & Apr 28,2003 & $9.08 \times 10^{-4}$ & $3.43 \times 10^{-2}$ & $4.31 \times 10^{-2}$ \\
\hline Manila & Oct 27,2003 & $2.38 \times 10^{-5}$ & $4.05 \times 10^{-3}$ & $3.84 \times 10^{-3}$ \\
\hline Lae & Oct 10,2003 & $1.89 \times 10^{-4}$ & $6.42 \times 10^{-3}$ & $7.68 \times 10^{-3}$ \\
\hline Nanyang & Oct 30, 2003 & $4.94 \times 10^{-4}$ & $2.90 \times 10^{-3}$ & $3.36 \times 10^{-3}$ \\
\hline
\end{tabular}

In TABLE $3, D_{4}$ is small for all days and stations, that means using either pseodurange as input, Reg-Est estimation results are very close to each other. Comparison with JPL results given as $D_{5}$ and $D_{6}$ are relatively small, therefore Reg-Est estimates are in good agreement with 2 hour TEC estimates of JPL.

\section{Effect of Ionospheric Thin Shell Height}

Slant Ray Total Electron Content (STEC) can be computed using Eq. (1). Vertical Total Electron Content (VTEC) can be computed as in Eq. (2) and using a thin shell approximation which is known as Single Layer Ionosphere Model (SLIM). In Eq. (3), $M$ is the mapping function and $\varepsilon$ is the local satellite elevation angle.

In SLIM model, ionosphere is assumed to be a layer of infinitesimal thickness. Ionospheric shell height is the height of maximum electron density and it is a function of time and geographic location [4]. Various methods in the literature have different ionospheric selection choices such as [1], [4], [6], [11], [14]. In [4], choosing different ionospheric heights can result TEC differences at 2 TECU level. In Fig. 3, Reg-Est estimates for $300 \mathrm{~km}, 428.8 \mathrm{~km}$ and $450 \mathrm{~km}$ are given for an example scenario. In Fig. 3, it is seen that TEC estimation results are very close to each other. To obtain a quantitative measure for the difference between TEC estimates, the following differences are defined.

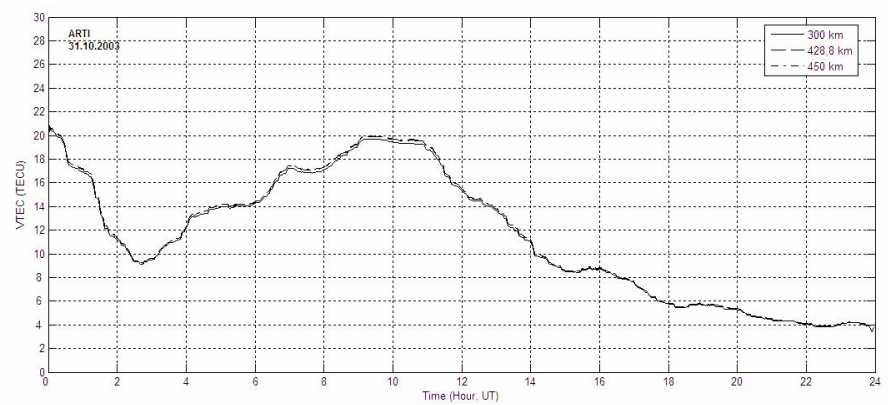

Fig. 3. Reg-Est TEC estimates for $300 \mathrm{~km}, 428.8 \mathrm{~km}$ and $450 \mathrm{~km}$ ionospheric shell height, Arti 31.10.2003, negatively disturbed day.

$D_{7}=\frac{1}{N} \sum_{n=1}^{N}\left|\boldsymbol{x}_{h 2}-\boldsymbol{x}_{h 1}\right|$

$D_{8}=\frac{1}{N} \sum_{n=1}^{N}\left|\boldsymbol{x}_{h 3}-\boldsymbol{x}_{h 2}\right|$

In Eq. (14) and Eq. (15), the mean TEC differences are given. $\boldsymbol{x}_{h 1}, \boldsymbol{x}_{h 2}$, and $\boldsymbol{x}_{h 3}$ are the Reg-Est TEC estimation results for 300 $\mathrm{km}, 428.8 \mathrm{~km}$ and $450 \mathrm{~km}$, respectively. $N$ is the total number of GPS recordings for 24 hour period. The differences are shown for various days and stations in TABLE 4. In TABLE 4, all TEC differences are below 1 TECU level. Thus, it can be concluded that the choice of the ionospheric shell height does not have any significant effect on Reg-Est TEC estimates. Thus, in IONOLAB, the height of $428.8 \mathrm{~km}$ can be used.

TABLE 4

Reg-Est TEC estimation differences with respect to ionospheric height

\begin{tabular}{|c|c|c|c|}
\hline Receiver Station & Day & $\mathrm{D}_{7}$ & $\mathrm{D}_{8}$ \\
\hline Ankara & Oct 10,2003 & 0.244 & 0.038 \\
\hline Ankara & Oct 31,2003 & 0.286 & 0.044 \\
\hline Zelenchukskaya & Oct 10,2003 & 0.207 & 0.032 \\
\hline Zelenchukskaya & Oct 28,2003 & 0.303 & 0.047 \\
\hline Arti & Oct 10,2003 & 0.162 & 0.025 \\
\hline Arti & Oct 31,2003 & 0.143 & 0.022 \\
\hline Metsahovi & Oct 10,2003 & 0.168 & 0.026 \\
\hline Metsahovi & Oct 28,2003 & 0.214 & 0.033 \\
\hline Nanyang & Oct 10,2003 & 0.534 & 0.083 \\
\hline Nanyang & Oct 30,2003 & 0.374 & 0.058 \\
\hline
\end{tabular}

\section{Weighting GPS measurements}

Signals from satellites of low elevation angles are more susceptible to multipath effects compared to signals from satellites of high elevation angles [13]. Therefore, it is important to apply an appropriate weighting procedure to minimize the multipath effects. In some studies, measurements obtained from satellites that are below a certain elevation angle limit are ignored. In $[6], \operatorname{an}^{2}\left(\varepsilon_{m}\right)$ is used as a weighting function where $\varepsilon_{m}$ is the satellite elevation angle. In this study, three different weighting options are tried for the Reg-Est method. These weighting options are given below. 
1. Weighting Function :

$$
w 1_{m}(n)= \begin{cases}0, & \varepsilon_{m}(n) \leq 10^{\circ} \\ \exp \left(-\left(90-\varepsilon_{m}(n)\right)^{2} / 2 \sigma^{2}\right), & 10^{\circ} \leq \varepsilon_{m}(n) \leq 60^{\circ} \\ 1, & 60^{\circ} \leq \varepsilon_{m}(n) \leq 90^{\circ}\end{cases}
$$

2. Weighting Function :

$$
w 2_{m}(n)= \begin{cases}0, & \varepsilon_{m}(n) \leq 10^{\circ} \\ \exp \left(-\left(60-\varepsilon_{m}(n)\right)^{2} / 2 \sigma^{2}\right), & 10^{\circ} \leq \varepsilon_{m}(n) \leq 60^{\circ} \\ 1, & 60^{\circ} \leq \varepsilon_{m}(n) \leq 90^{\circ}\end{cases}
$$

3. Weighting Function :

$w 3_{m}(n)=\sin ^{2}\left(\varepsilon_{m}(n)\right)$

The first weighting function is the one that is used previously in Reg-Est. This function ignores the measurements below $10^{\circ}$ elevation angle. The measurements between $10^{\circ}$ and $60^{\circ}$ are weighted using a Gaussian function which has a mean at $90^{\circ}$. The measurements above $60^{\circ}$ are directly used. The second weighting function is similar to first one except the gaussian function has a mean at $60^{\circ}$. The third weighting function is the one that is used in [6]. These weighting options are tried in Reg-Est method for various days and receiver stations that are listed in TABLE 1. An example is provided in Fig. 4 and TEC estimates for Ankara 10.10.2003 using w1, w2 and $w 3$ are given. In Fig. 4, the estimates obtained by $w 2$ and $w 3$ weighting functions are close to each other. These two functions provide smooth transitions in time compared to those of $w 1$. In order to examine the TEC estimates in detail, the following normalized difference functions are defined. The normalized differences obtained using these three difference functions are given in TABLE 5 for some stations and days as an example.

$$
\begin{aligned}
& \mathrm{D}_{9}=\frac{1}{N} \sum_{n=1}^{N} \frac{\left|\boldsymbol{x}_{w 3}-\boldsymbol{x}_{w 2}\right|^{2}}{\left|\boldsymbol{x}_{w 2}\right|^{2}} \\
& D_{10}=\frac{1}{N} \sum_{n=1}^{N} \frac{\left|\boldsymbol{x}_{w 2}-\boldsymbol{x}_{w 1}\right|^{2}}{\left|\boldsymbol{x}_{w 2}\right|^{2}} \\
& D_{11}=\frac{1}{N} \sum_{n=1}^{N} \frac{\left|\boldsymbol{x}_{w 3}-\boldsymbol{x}_{w 1}\right|^{2}}{\left|\boldsymbol{x}_{w 2}\right|^{2}}
\end{aligned}
$$

In TABLE $5, D_{9}$ values are smaller than $D_{10}$ and $D_{11}$ which shows that TEC estimation results of $w 2$ and $w 3$ are in relatively better accordance for all stations compared to results of $w 1$. Since $w 2$ provides smooth transitions and reduces sudden irregularities in TEC estimates, $w 2$ can be used in IONOLAB.

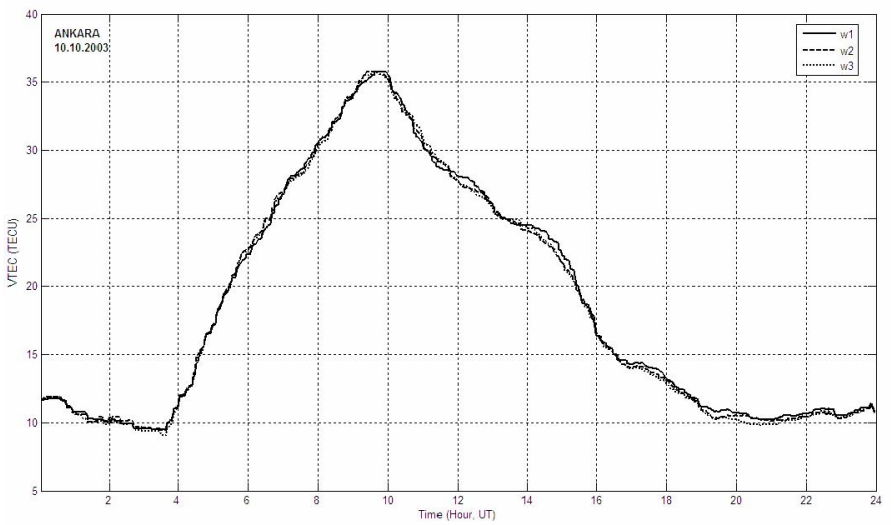

Fig. 4. Reg-Est TEC estimates obtained w1, w2 and w3 weighting functions for Ankara 10.10.2003.

TABLE 5

Reg-Est TEC estimation differences with respect to weighting functions w1, $\mathrm{w} 2$ and $\mathrm{w} 3$.

\begin{tabular}{|c|c|c|c|c|}
\hline Receiver Station & Day & $D_{9}$ & $D_{10}$ & $D_{11}$ \\
\hline Ankara & Oct 10,2003 & $9.51 \times 10^{-5}$ & $2.34 \times 10^{-4}$ & $3.06 \times 10^{-4}$ \\
\hline Ankara & Oct 31,2003 & $1.20 \times 10^{-4}$ & $2.34 \times 10^{-4}$ & $3.31 \times 10^{-4}$ \\
\hline Zelenchukskaya & Oct 10,2003 & $8.69 \times 10^{-5}$ & $4.03 \times 10^{-4}$ & $4.34 \times 10^{-4}$ \\
\hline Zelenchukskaya & Oct 28,2003 & $5.14 \times 10^{-5}$ & $1.92 \times 10^{-4}$ & $1.68 \times 10^{-4}$ \\
\hline Arti & Oct 10,2003 & $7.77 \times 10^{-5}$ & $4.09 \times 10^{-4}$ & $3.42 \times 10^{-4}$ \\
\hline Arti & Oct 31,2003 & $2.67 \times 10^{-4}$ & $8.72 \times 10^{-4}$ & $8.36 \times 10^{-4}$ \\
\hline Metsahovi & Oct 10,2003 & $1.53 \times 10^{-4}$ & $5.06 \times 10^{-4}$ & $5.95 \times 10^{-4}$ \\
\hline Metsahovi & Oct 28,2003 & $1.62 \times 10^{-4}$ & $3.04 \times 10^{-4}$ & $5.20 \times 10^{-4}$ \\
\hline Nanyang & Oct 10,2003 & $1.71 \times 10^{-4}$ & $7.39 \times 10^{-4}$ & $9.41 \times 10^{-4}$ \\
\hline Nanyang & Oct 30,2003 & $2.78 \times 10^{-4}$ & $6.98 \times 10^{-4}$ & $1.40 \times 10^{-3}$ \\
\hline
\end{tabular}

\section{CONCLUSION}

Reg-Est, developed in [1], [2], and [3], is a high resolution, robust TEC estimation technique. In this paper, the use of satellite and receiver biases, the effects of ionospheric shell height and the choice of weighting functions are investigated for further improvement of Reg-Est. Although there is no standard way of using satellite and receiver instrumental biases in the literature, two methods for adding these biases is applied and the results are compared with IGS analysis centers. The results are consistent with IGS centers especially with JPL and CODE. The method which estimates TEC closest to IONEX estimates for the use the instrumental biases is selected for IONOLAB. In previous studies of RegEst, only pseudo-range measurement were used as input to the regularization algorithm. In this paper, phase measurements are used in Reg-Est method with an appropriate leveling technique. The TEC estimation results are very close to the results of pseudorange measurements but TEC estimates from phase-leveled measurements are less noisy.

Ionospheric shell height is a parameter used in RegEst. In this paper, different ionospheric height values are used in Reg-Est method and the TEC estimates are compared. It is observed that the Reg-Est method is nearly independent of the choice of ionospheric height. Weighting function helps to reduce the multipath effect in the measurements of satellites which are at low elevation angles. Three different weighting 
options are tried and the weighting function which reduces the non-ionospheric effects best is selected for IONOLAB. It is also shown that the TEC estimation results of IONOLAB is consistent with IGS analysis centers especially with CODE and JPL. .

\section{ACKNOWLEDGMENT}

This study is supported by TUBITAK EEEAG grant no: $105 \mathrm{E} 171$.

\section{REFERENCES}

[1] Arıkan, F., Erol, C.B., Arıkan, O., "Regularized Estimation of Vertical Total Electron Content from Global Positioning System Data", Journal of Geophysical Research, (118) 1469-1480, 2003.

[2] Arıkan, F., Erol, C.B., Arıkan, O., "Regularized Estimation of Vertical Total Electron Content from GPS Data for a Desired Time Period", Radio Science, 39:RS6012, 2004

[3] Arıkan, F., Erol, C.B., Arıkan, O., "Regularized Estimation of TEC from GPS Data for Certain Midlatitude Stations and Comparisons with IRI Model", J. Adv. Space Res., doi:10.1016/j.asr.2007.01.082, 2007.

[4] A. Komjathy., R.B. Langley, "An assessment of predicted and measured ionospheric total electron content using a regional GPS Network, ION National Technical Meeting", Santa Monica, CA, 22-24 January 1996.

[5] E. Calais., B. Minster, "GPS, earthquakes, the ionosphere and the space shuttle", Physies of the Earth and Planetary Interiors, ION National Technical Meeting, 105, 167-171, 1998.

[6] G. Ma, T. Maruyama, "Derivation of TEC and estimation of instrumental biases from GEONET in Japan", Annales Geophysicae, (21) 2083-2093, 2003.

[7] G. E. Lanyi, T. Roth, "A comparison of mapped and measured total ionospheric electron content using Global Positioning System and beacon satellite observations", Radio Sci., 23, 483-492, 1998.

[8] Ionospheric Dispatch Center. http://www.cbk.waw.pl/rwe/q_d_days.ctl

[9] N. Jakowski., "Generation of TEC maps over COST251 area based on GPS measurements", Proc. of the 2nd COST 251 Workshop, Side, 30-31 March, 51-57, 1998.

[10] R. Warnant, Reliability of the TEC computed using GPS measurements: The problem of hardware biases, Acta Geod. Geoph. Hung., 32 (3-4), 451-459, 1997

[11] S. Schaer, "Mapping and Predicting the Earth's Ionosphere Using the Global Positioning System", Ph.D. thesis, University of Bern, Bern, 1999.

[12] TEC maps of IGS analysis centers in Ionex format. ftp://cddisa.gsfc.nasa.gov/gps/products/ionex/

[13] X. Liao, "Carrier phase based ionosphere recovery over a regional area GPS network", M.S. Thesis, University of Calgary, Calgary, 2000.

[14] Y. Otsuka, T. Ogawa, A. Saito, T. Tsugawa, S. Fukao, S. Miyazaki, "A new technique for mapping of total electron content using GPS network in Japan", Earth Planets Space, (54) 63-70, 2002. 